\title{
Le doigt d'Oreste
}

Orestes' finger

\section{Marcel Detienne}

\section{Q OpenEdition}

\section{Journals}

\section{Édition électronique}

URL : http://journals.openedition.org/span/1450

DOI : $10.4000 /$ span. 1450

ISSN : 2268-1558

\section{Éditeur}

École pratique des hautes études. Sciences humaines

\section{Édition imprimée}

Date de publication : 1 août 1996

Pagination : 23-38

ISSN : 0294-7080

\section{Référence électronique}

Marcel Detienne, «Le doigt d'Oreste », Systèmes de pensée en Afrique noire [En ligne], 14 | 1996, mis en ligne le 17 avril 2014, consulté le 09 octobre 2020. URL : http://journals.openedition.org/span/1450 ; DOl : https://doi.org/10.4000/span. 1450 


\title{
LE DOIGT D'ORESTE
}

\author{
par
}

\section{Marcel Detienne}

En ouverture, deux figures de meurtrier grec (Parker, 1983). L'une, furtive, à la fin de l'lliade ; l'autre, massive et violente, aux commencements d'une cité. Hector vient d'être tué, son cadavre est souillé, horriblement maltraité ; chaque jour, Achille le traîne trois fois autour de Troie, ses chevaux lancés au grand galop. Un soir, le père d'Hector, Priam, ne peut plus supporter le spectacle, sa folle violence, sa démesure sacrilège. Guidé par Hermès, il pénètre dans le camp des ennemis de Troie, il s'approche d'Achille, lui embrasse les genoux, lui baise les mains, des mains terribles, "meurtrières" (androphónoi), ces mains qui lui ont tué tant de fils. "Ainsi quand une lourde erreur (atè) a fait sa proie d'un mortel et qu'après être devenu un meurtrier (katakteinas) dans son pays (patrè), il arrive en terre étrangère, un logis d'un homme opulent, la stupeur (thambos) saisit tous ceux qui le voient. Même stupeur saisit Achille à voir Priam semblable aux dieux, même stupeur prend les autres..." (Iliade, XXIV, 477-483). L'inconnu, surgi de la nuit et qui fait les gestes de la supplication, est le père du cadavre outragé, jeté aux pieds d'Achille, mais il ressemble à un de ces fugitifs qui, pour avoir versé le sang dans sa "patrie", s'en va, s'exile en quête d'un foyer et d'une maison qui l'accueillera, lui

Destins de meurtriers

Systèmes de pensée en Afrique noire, 14, 1996 
fera place en lui offrant un nouveau statut, loin de l'erreur et du sang répandu. Devant Priam, l'homme qui lui a tué ses enfants, Achille et ses mains "meurtrières" (androphónoi) : qui font couler le sang humain mais celui des guerriers, dans l'espace de la mort rouge et de la guerre ouverte.

Seconde figure : celle des Alcméonides, une maison de plusieurs centaines de personnes dans l'Athènes archaïque (Moulinier, 1952 : 46-47, et Durand, 1990 : 276-278). Vers 650 av. notre ère, un peu avant que Dracon ne propose les premières lois sur l'homicide, les partis s'affrontent et la cité se déchire. Un athénien, nommé Cylon, s'empare de l'Acropole, ses adversaires l'y assiègent. La cité est coupée en deux. Les Alcméonides promettent aux partisans de Cylon la vie sauve, s'ils quittent aussitôt l'Attique. Épuisés, mourant de soif et de faim, les Cylonides, constitués en suppliants d'Athéna, refusent de quitter la protection de la déesse. Un fil de laine, attaché à la statue, doit leur assurer dans leur descente le contact avec la puissance protectrice. A proximité de l'Aréopage - qui va devenir un des grands tribunaux du sang - , le fil casse, les Cylonides se précipitent sur l'autel des Euménides : ils sont égorgés sur le champ par les Alcméonides et leurs alliés. Fléau (loimos), souillure, miasma: frayeurs (phoboi), apparitions inquiétantes (phasmata), malformations des victimes sacrificielles, la cité entière est gangrenée. Les Alcméonides sont déclarés Impurs, Enagêts, "enclos dans la souillure". Ils sont qualifiés d'Alitèrioi de la déesse, sacrilèges habités par la colère des morts criant vengeance. Il faut les expulser, tous et même leurs morts, il faut les exhumer, rejeter les ossements hors des limites du territoire, de la chôra, de la cité. Il faudra donc faire appel à un purificateur hors pair, venu de Crète et appelé Epiménide qui procédera à une véritable refondation du territoire à travers ses autels et ses sanctuaires.

Deux scénarios des effets du sang versé qui, malgré leurs différences, partagent le recours à la fondation - refondation pour mettre fin à la souillure. Deux scénarios qui laissent en creux le statut de l'homicide et les institutions de la cité pour répondre au sang versé, mais le premier, en montrant les mains "meurtrieres" d'Achille, conduit vers le nom technique de l'homicide, tandis que le second découvre sur la scène du drame le tribunal de l'Aréopage et les 
puissances dites "les bienveillantes", Euménides. Pour les cités grecques, en regard des sociétés riches en rituels encadrant le meurtrier et sa victime, le fait majeur, c'est l'apparition de tribunaux du sang, d'un droit pénal en matière d'homicide (Van Effenterre \& Ruzé, 1994). Les premières cités légifèrent d'abord sur le sang versé, sur le meurtre d'un homme du même groupe, de cette communauté nouvelle de ceux qui appartiennent à une même cité. Entre 620 et 530, approximativement, les lois, les thesmoi sur le meurtre s'écrivent en lettres hautes et colorées sur des stèles dressées aussi bien par de jeunes cités en Sicile et en Grande Grèce que dans des cités plus installées du continent comme Argos ou Athènes. L'homicide est réglementé par la cité : si un individu est tué dans l'espace de la cité, c'est désormais la cité (polis) qui se sent atteinte, c'est elle qui fixe la réparation due aux parents et à la collectivité. "La solidarité civique joue par-dessus la discipline de la famille ou la protection d'un patron", ainsi que l'ecrit Louis Gernet (1955 et 1986). Le "meurtre" devient une catégorie juridique; le meurtrier, appelé androphónos - comme la main d'Achille ou comme le guerrier homérique -, devient avec Dracon un sujet de droit. La loi sur l'homicide de Dracon - ce qui s'appelle alors andrephonikos tethmos - s'applique de manière impartiale à différencier les catégories de fautes, à doser la culpabilité, à marquer des degrés, et à instituer des tribunaux différents pour les juger. On va ainsi distinguer le meurtre, phónos, volontaire, involontaire, prémédité, le meurtre dit légitime, juste, dikaios, dira-t-on plus tard. Tout un droit avec action publique et tribunaux populaires va s'édifier autour de la notion d'agent de celui qui agit, ho drasas : notion abstraite autant que celle de citoyen. On va analyser les intentions de cet "agent", mesurer sa responsabilité, apprécier ses degrés d'engagement. Parallelement, la cité va instituer un espace de jugement, de l'affrontement duel entre le présumé coupable et l'accusation : un champ clos où les parties en présence sont définies par l'instance nouvelle du juridique.

Autre nouveauté des cités grecques : l'espace de la guerre. Lui aussi reçoit son autonomie. La cité fait de la guerre une affaire publique, conduite par ses magistrats, accomplie par les citoyens en armes. Une guerre se décide en assemblée ; elle se déroule dans un 
espace choisi, souvent propice au choc frontal de deux phalanges, chargeant l'une contre l'autre. Il n'y a plus de place pour la razzia, ni pour la vendetta, la vengeance privée, l'expédition des covengeurs d'une même maison contre une autre. Le sang versé à la guerre n'est pas un homicide, il n'entraîne ni souillure, ni purification. C'est d'ailleurs une des caractéristiques des lois de Dracon : il n'y est pas question de purification, pas plus que de souillure en général. En devenant sujet de droit, le meurtrier semble se dépouiller de la ritualité dont l'entourent volontiers des cultures marquées par le même archaïsme, dans l'Afrique de l'Ouest ou dans le monde amérindien. En l'occurrence, et dans la perspective comparatiste qui est ici la nôtre, les cités grecques, tout en portant témoignage d'une rupture et d'un commencement radical, font voir parallèlement la prégnance de représentations socio-religieuses du meurtrier et de la souillure entre le VIIeme et le IVème siècles avant notre ère : déchaînement de puissances anonymes éveillées par la souillure d'un groupe, sinon d'un territoire ; maladies effroyables montant à l'assaut des chairs du meurtrier; purifications par le sang des victimes coulant sur le corps de l'homicide. Autant de figures que l'historien, attentif aux changements, est tenté de rassembler afin de les assigner à un état social et religieux antérieur à l'avènement de la cité et, plus précisément, à l'entrée dans le droit explicite des tribunaux du sang.

Aujourd'hui, nous hésitons à reconnaître à l'horizon de la ou des cités grecques une forme de pensée mythico-religieuse qui serait marquée par ce que Louis Gernet appelait, en 1949, le prédroit, pensée globale habitée par le mythe et qui allait s'effacer devant la rationalité du juridico-politique ( $c f$. Detienne, 1994). Nous ne pouvons pas davantage croire avec Marie Delcourt, écrivant en 1959 un remarquable essai sur le matricide et sa projection légendaire en Grèce, que "l'ethnologie délimitera un jour synchroniquement et diachroniquement l'aire d'extension de ... croyances" (Delcourt, 1959), comme celles qui proliferent dans le sillage d'Oreste, meurtrier de sa mère pour être vengeur de son père. C'est précisément aux entours de l'extrême assassin Oreste que se sont fixées, dans la tradition grecque, les représentations à la fois les plus vives et les plus diverses des effets du sang verse : folie du meurtrier, identification avec la victime, fuite, exil, 
purifications répétées par le sang, fondations d'autels et de sanctuaires, jugements devant les tribunaux qui ne peuvent mettre un terme aux errances ni à l'envahissement du meurtrier par les puissances du sang répandu. Trois quarts de siècle après Dracon, et pendant une période comprise entre 550 et 400 , le matricide Oreste propose la configuration la plus riche des liens qui peuvent se nouer entre l'homicide et celui ou celle dont le sang a été versé. Dans la culture grecque, c'est le dossier "Oreste" qui offre au comparatiste anthropologue les données le mieux articulées pour analyser les effets du meurtre dans le corps du meurtrier et pour mettre ainsi en perspective une série de sociétés qui font une place de choix au meurtrier et à son éclatante souillure.

A la singularité d'Oreste, il faut un contexte, et double : d'une part, les modalités de transmission de la souillure en pays grec ; de l'autre, les effets réguliers du sang versé sur le meurtrier demeurant sur les lieux mêmes du crime. Premier contexte entrevu dans l'affaire des Alcméonides : le meurtre des Cylonides suppliants cramponnés à l'autel des Euménides qui sont l'autre face des Erinyes, les comptables du sang versé sur le territoire d'Athènes, déclenche une souillure en forme de raz-de-marée : elle submerge l'ensemble du groupe, de la maison des Alcméonides (on parlera de 700 personnes), elle attaque la totalité des autels et des sanctuaires du territoire de l'Attique, elle atteint si profondément les racines de ces présumés autochtones qu'Epiménide, le purificateur appelé de Crète pour endiguer le fléau, le loimos, devra se livrer à une refondation des liens avec la terre en édifiant des autels et en sacrifiant des victimes animales à l'endroit où elles choisissent de se coucher, désignant ainsi comme dans les rituels de fondation des cités nouvelles le lieu adéquat aux puissances du terroir. "A souillure majeure, il faut un purificateur absolu", comme l'écrit Jean-Louis Durand (1990 : 276). Au Vème siècle, les médecins hippocratiques, écartant de leurs analyses les maladies du type "fléau" ou loimos, refusent le modele d'une souillure qui pourrait atteindre tous les membres d'une même communauté : ils défendent la thèse du contact singulier de chacun avec le facteur de maladie. Le corps malade ou "souillé" d'un individu est appréhendé, très tôt, en dehors de sa relation avec un territoire, et même à l'écart de son appartenance à une communauté politique. Dans ce milieu de savoir comme dans la 
pensée juridique de Dracon, et un peu plus tôt, toute représentation d'un champ de souillure emplissant la totalité d'un territoire est exclue autant que celle de purification de caractère religieux. Au modèle mythico-religieux de l'affaire des Alcméonides le milieu des tribunaux du sang va substituer un paradigme de type politique qui va sévir entre Dracon et la cité grecque du IVème siècle avant notre ère. Le meurtrier doit être tenu à l'écart d'une série de points sensibles de l'espace de la cité : de l'eau lustrale ; il ne doit pas s'approcher des vasques placées à l'entrée des sanctuaires et de l'agora; des libations quelles qu'elles soient faites en l'honneur des dieux; des cratères, ceux des salles de banquet ou qui sont dressés parfois dans les rues; des sanctuaires, des autels, de tous les lieux consacrés aux relations privilégiées entre les dieux et les hommes. Des listes semblables se retrouvent entre Eschyle, Platon et Antiphon: un meurtrier ne peut plus habiter la maison de son père, ni sacrifier sur les autels de son dème, ni prendre l'eau lustrale dans sa phratrie ; il sera tenu à l'écart des nomima, des lieux et des moments religieux traditionnels ; il ne doit "souiller", miainein, ni les sanctuaires, ni la place publique, ni les ports, ni aucun autre lieu de réunion : l'interdiction lui en est faite, publiquement et par déclaration solennelle. Le meurtrier est "excommunié" au sens politique ; il cesse d'avoir part, de participer à tout ce qui constitue la vie socio-politique des citoyens. Antiphon ou l'auteur des Tétralogies insiste sur la transmission de la souillure en parlant de "remplissage" : "le miasma remplit les innocents à la table desquels le coupable s'assied". Ana ou kata ou sunkata-pimplènai : remplir par excès, par saturation et singulièrement en ces lieux où les citoyens mangent et boivent ensemble, en ces lieux d'assemblées, de sacrifices, de réunions et de banquets, là où les citoyens forment une sorte d'organisme homogène. C'est là où se pratique le plus activement la similitude, l'homoiotès, que la souillure du meurtrier risque le plus de "remplir excessivement" la communauté politique. Plus que d'impureté religieuse, il convient de parler de souillure politique. Au milieu de toutes les vicissitudes de sa carrière de meurtrier, Oreste se verra contraint, en terre athénienne, de boire et de manger seul, sous le signe de la fête hivernale du vin nouveau! 
Autre contexte qui permet de prendre mesure des étrangetés d'Oreste : les effets du sang versé sur le corps et l'esprit du meurtrier. Les Alcméonides, souillés du sang des Cylonides, sont très tôt qualifiés d'enageîs, englobés dans la souillure, possédés, saisis par une puissance de souillure, une puissance le plus souvent anonyme et génétique, tantôt daimôn, tantôt Erinye. "Certain vieux récit de la tradition", évoqué par Platon dans les Lois (IX, 865 d.), témoigne que "l'homme mort de mort violente s'il a vécu libre et fier, est, sitôt mort, irrité contre celui qui l'a tué, et lui-même rempli de crainte et de frayeur à la suite de la violence subie, ne peut voir son propre meurtrier vivre et avoir les habitudes qui lui étaient à lui-même coutumières sans être pris d'effroi et, lui-même plein de trouble, sans troubler autant qu'il peut son meurtrier dont il prend la mémoire comme alliée pour l'inquiéter en son âme et dans ses actes. Ainsi faut-il que le meurtrier se retire devant sa victime pendant toutes les saisons successives d'une année et quitte tous les lieux où il était chez lui, en quelque endroit de sa patrie que ce puisse être". Représentations que n'abolit pas la législation sur le sang ni la délimitation d'un espace politique que ne peuvent plus partager le meurtrier et ses ex-concitoyens. Cette fois, il s'agit des liens obscurs et terribles qui viennent se nouer entre l'homicide et sa victime, entre le "nouveau mort" (neothnès) et son meurtrier. Mélange de colère, de désir et de peur affolée: être en colère, erinuein, ce verbe si actif dans le nom même des Erinyes, ces puissances éveillées par le sang versé ; être envahi par du thumos, être enthumios, inquiet, tourmenté par ce qu'il doit faire ou ce qu'il a fait ; être en proie à la peur, à l'effroi, à l'épouvante, être affolé et affoler lui-même de toute la force de son affolement son assassin. La violence subie invite à la violence en retour : le meurtrier est envahi par les puissances de peur et d'effroi qui agitent la victime quand elle est ce "nouveau mort", assiégeant celui qui a versé son sang, lui disputant des lieux familiers et habitant à chaque instant la mémoire de son assassin. Si le meurtrier ne veut pas sombrer dans la folie, sinon davantage, il faut qu'il s'exile, qu'il déserte les lieux fréquentés par sa victime et lui-même, afin d'échapper à la force d'une souillure - colère qui se tourne - "(prostropaïque)" - vers son meurtrier, jusqu'à la possession et à l'identification. 
Souillure et territoire, victime et meurtrier : traditions et représentations des Grecs méritent d'être confrontées à celles d'un certain nombre de sociétés amérindiennes ou africaines. Certaines d'entre elles mettent en relation étroite un type de souillure et un espace social à travers une puissance, souvent anonyme, qui "sanctionne la souillure survenant dans l'aire de sa juridiction". Un "champ de souillure", selon la formule de Michel Cartry et d'András Zempléni, semble pensé et défini comme un espace-corps englobant les corps de tous ceux qui lui appartiennent. La souillure, dans ce cas, est ressentie comme une entame faite à la plénitude du corps global. En guise de réparation ou de purification de la dite souillure, la puissance du corps entamé réclame que l'on restaure sa plénitude en retraçant ses limites. Purification ou réparation qui prend la forme d'un encapsulage, d'un "enkystage", disaient les mêmes africanistes, par exemple du meurtrier à l'intérieur du groupe ou du segment d'espace contaminé. Sans ouvrir la comparaison sur ce point, d'emblée, surgissent une série de différences avec le monde grec : aucune souillure sexuelle n'est assimilable à celle du sang versé, pas même celle de l'inceste qui, d'ailleurs, n'est pas en Grèce un vrai miasma ; l'espace social d'un lignage ou d'un autel de la terre n'est pas homologue à l'espace politique de ces groupes de solidarité qui ponctuent le domaine de la cité ; à l'entame répond la saturation, tandis que la redéfinition des limites par "enkystage" du meurtrier s'oppose à la manière grecque d'expulser l'homicide, invité ainsi à se tracer un espace neuf à l'écart de ses lieux familiers. Dans une série de traditions d'ordre mythique, l'auteur d'un meurtre se voit valorisé, non pas à l'intérieur même de sa société, mais dans l'espace ouvert et étranger que les cités grecques pensent volontiers comme vierge, en friche et sans appartenance aucune.

Ce sont les procès d'identification du meurtrier et de la victime qui semblent offrir ici les éléments les plus intéressants pour une mise en perspective des sociétés anciennes et de certaines sociétés africaines et amérindiennes. Au départ, Oreste semble fort étranger à l'image du matricide qui va le marquer entre Stésichore d'Himère en Grande Grèce et Euripide d'Athènes en son thêtre. Dans l'Odyssée, Oreste qui veut venger son père tue Egisthe ainsi que Clytemnestre. Athena fait son éloge en face de Télémaque, tandis qu'Oreste, à Argos même, 
sur place, célèbre les funérailles de ses deux victimes, offrant le banquet funèbre à l'ensemble des Argiens (Odyssée, I, 30; 298-300 et III, 309). Il n'y a pas de matricide. Rien qu'un vengeur. Ni purification, ni tribunal. La gloire que vaut à Oreste d'avoir vengé son père éclaire le destin de Télémaque et fait signe en direction de la vengeance qui est la part intouchable d'Ulysse faisant retour. Milieu du Vlème siècle avant notre ère : un autre Oreste s'enfuit dans la nuit, échappant à la fureur meurtrière de Clytemnestre (version de Stésichore: PMG, Fr. 215-219. Cf aussi Neschke, 1986: 283-301). C'était quelque part en Laconie. Emmené a Delphes, Oreste reçoit d'Apollon l'ordre de verser le sang de sa mère et de l'amant de Clytemnestre. Le dieu de Delphes remet au vengeur son arc, un arc qui a fait ses preuves : les fleches meurtrières tiendront en respect l'Erinye surgie du sang fraîchement versé de la mère d'Oreste. L'Apollon de Delphes connaît la souillure, l'exil et l'épouvante. Naguère, en Argolide, après le meurtre de Python, il fut saisi de si grandes frayeurs - en un lieu appelé "aujourd'hui encore" Terreur qu'il s'enfuit jusqu'en Crète vers les seuls purificateurs experts en de si hautes souillures. Apollon connaît les chemins d'Oreste, dieu meurtrier, exilé et même impur, il sait intimement, au milieu des meurtriers montant vers sa demeure à Delphes, la possession horrifiée de l'assassin par sa victime se dressant soudainement et sifflant de colere (Pausanias, II, 7, 7-9).

Ce dieu impur et meurtrier, Pindare, en 474 av. notre ère, lui donnera le nom d'Arès, le fou guerrier, le maître emporté par la violence des batailles et des tueries (Pindare, Pythique, IX, 36-37). Il sera encore Apollon, "le pur exilé du ciel", dans l'Orestie d'Eschyle, un peu plus tard, quand Oreste fera en la compagnie du dieu l'expérience cumulée de l'exil, pour user sa souillure au long des chemins, de la purification, pour que le sang d'une victime animale le lave du meurtre d'une mère, et, enfin, du tribunal humain de l'Aréopage, de la colline d'Arès, pour retrouver une place dans la cité des hommes.

Autour d'Oreste, un ensemble de récits dispersés dans le Péloponnèse racontent les troubles du meurtrier, les attaques dont son corps est l'objet, les traitements que des rites appropriés lui font subir. En Achaïe, à Kerynaia, on montre un sanctuaire dit des Euménides, fondé 
par Oreste en ses errances. C'est un espace inquiétant même sous le nom apaisé des Erinyes: "Quiconque y pénètre en étant possédé par le sang versé ou une autre souillure... est aussitôt assailli par des visions effrayantes et se trouve hors de lui". Chambre des folies et des terreurs qui se sont abattues sur Oreste, la nuit même de la mort de Clyternnestre, quand le fils meurtrier s'est mis à bondir comme un possédé de Dionysos, assailli par la vision hallucinée des "Trois Vierges, semblables à la nuit" (Pausanias, VII, 25,7). En Arcadie, sur la route de Mégalopolis à Messène, il est un autre lieu où est passé Oreste (Pausanias, VIII, 34, 1-3). On l'appelle Les Furies ainsi que le pays alentour. C'est là qu'Oreste est entré en fureur et que, saisi de folie, possédé par les Maniai, il a dévoré un morceau de sa main, un doigt arraché que commémore un léger tumulus dit le "Monument du Doigt", à deux pas du sanctuaire en l'honneur des Furies. En regard de ce premier sanctuaire, appelé Guérison $(A k \grave{e})$, qui est aussi un sanctuaire consacré aux Euménides, l'autre face des violentes et furieuses Erinyes. Au moment où les Furies l'envahissaient, Oreste avait vu leurs faces noires. Une fois amputé de ce fragment de lui-même, les mêmes puissances lui étaient apparues blanches et lumineuses. A ce spectacle, Oreste aurait retrouvé la raison, et, pour mieux l'assurer, il aurait offert sur place deux sacrifices sanglants : l'un de type expiatoire (enagidzein) aux Déesses Noires pour écarter leur colère; l'autre de nature alimentaire à l'adresse des Puissances Blanches, en l'occurrence conviées en même temps que les Grâces Charites. Un dernier sacrifice dit "de la chevelure coupée" était venu sanctionner le terme de l'égarement et le retour à soi : sacrifice du Coureion, lorsqu'Oreste avait coupé sa chevelure d'égaré hirsute, comme s'il renaissait, devenait un de ces jeunes hommes dont l'entrée dans l'âge adulte était marquée à Athènes par un sacrifice appelé Coureion, de la tonte, celle des moutons et des chèvres offerts en sacrifice pour les jeunes garçons et leur première barbe à la puberté.

D'affreux malheurs menaçaient Oreste. Delphes en avait prophétisé la montée : "des maladies effroyables se jetant à l'assaut des chairs, des lèpres à la dent sauvage qui vont ravageant ce qui, la veille, était un corps, tandis que des poils blancs se lèvent sur les plaies" (Eschyle, Choéphores, 278-284. Delcourt, 1959 : 28-29). Qu'il ne 
venge pas son père ou qu'il verse le sang de sa mère, Oreste également assailli éprouve dans son corps la mort violente de l'autre : tantôt mort vivant, tantôt voué à l'auto-cannibalisme et semblant offrir à la victime faisant corps avec lui un morceau de sa propre chair, avant de pouvoir faire retour vers le sacrifice socialisé de l'autel et des victimes à nouveau partagées. A Trézène, en Argolide, c'est un Oreste durement éprouvé qui est soumis à une longue purification (Pausanias, II, 31, 4 \& 8-9). Neuf citoyens s'emploient à le débarrasser de la souillure du sang versé. Durant le traitement, Oreste est logé dans une baraque construite en face du sanctuaire d'Apollon car personne ne veut le recevoir dans sa maison! La purification semble se faire en deux temps : sur la pierre sacrée, dressée devant le temple d'Artémis ; dans la cabane isolée, où les purificateurs le nourrissent jusqu'au moment où il cesse d'être impur. Le régime alimentaire du meurtrier est un aspect important du traitement car aujourd'hui encore, note Pausanias, les descendants de ceux qui ont purifié Oreste y prennent leurs repas, à des jours déterminés. Non loin de la baraque dite d'Oreste, on enfouit tout ce qui a servi à la purification : restes de victimes, médecines diverses, car les neuf purificateurs ont eu recours, dit-on, à divers procédés, et en particulier à une eau provenant de la Fontaine du cheval, une eau associée à la puissance d'un cheval né de la terre, et voisine de l'eau terrible de Styx, Puissance de serment, coulant en cercle dans les entrailles de Gaia.

Davantage que l'eau, même dotée de si grands pouvoirs, le sang coulant sur le meurtrier a vertu pour le purifier de la souillure du sang répandu. "Ils veulent se purifier eux qui sont souillés de sang avec le sang d'un autre" (Héraclite, F.5 Bollach-Wissman). Héraclite en son ironie n'a pas besoin d'évoquer Oreste. D'autres meurtriers ou criminels possédés par une souillure connaissent la purification par le sang que certains "charlatans", dénoncés par les médecins hippocratiques, recommandent dans le traitement des " épileptiques", atteints de la maladie dite sacrée (Hippocrate, Sur la maladie sacrée, 4). Mais le matricide de l'Orestie raconte lui-même comment il a été purifié par Apollon, à Delphes dans le sanctuaire du dieu dit Phoibos, c'est-à-dire le Pur. "Ma souillure était fraîche quand au foyer de Phoibos l'offrande purificatrice d'un pourceau égorgé l'a chassée loin de moi" 
(Eschyle, Euménides, 280-285). Apollon fait office de purificateur, sur un de ses autels, peut-être celui réservé à Hestia, la puissance du feu domestique et sacrificiel installée dans la chambre, le mégaron d'Apollon. A la manière de celui qui purifie par le sang, le dieu fait couler sur la tête et sur les épaules d'Oreste le sang d'un porcelet, la victime la plus généreuse de ce liquide vital. Sur un cratère du Musée du Louvre, trouvé à Armento, Oreste assis sur l'autel d'Apollon reçoit le sang de la victime tenue au-dessus de lui, comme s'il était lui-même un autel vivant éclaboussé par la vie rouge de l'animal égorgé (Delcourt, 1959 : fig. 7, pp. 94-95).

Plus attentive que d'autres à ces procédures de régénération du meurtrier, déjà transformé par l'exil en mort vivant, Marie Delcourt suggérait d'y voir une posture identifiant l'homicide au nouveau-né sous l'organe ensanglanté, appelé en grec delphús, si proche du nom du cochon de lait, delphaks. Elle montrait même que certains vivants, considérés comme morts à la suite d'une disparition ou de funérailles accomplies par erreur, étaient soumis à un rituel circonstancié de renaissance (1959: 95-102). Sans exclure un symbolisme explicite de la nouvelle naissance, peut-être faut-il mettre en avant le traitement sacrificiel que le rituel de Trézène formule en termes choisis: la commensalité commémorative des purificateurs et de leurs descendants venant confirmer le régime alimentaire réservé à l'homicide enclos dans son cabanon. L'autel redoublé par la scène de purification avec le sang sacrificiel va dans le même sens : le meurtrier, si violemment exclu du cercle de ceux qui ont part au sacrifice en tant que rituel social et politique en Grèce, revient vers le sacrificiel, certes en fondant ici et là un autel, en offrant lui-même les sacrifices distincts qui vise à séparer les Euménides des Erinyes, mais, aussi plus radicalement, en tenant le rôle d'un autel que fonderait le sang versé d'une victime par un dieu comme Apollon, si volontiers fondateur de sacrifices, d'autels et de sanctuaires. C'est sur le même horizon sacrificiel qu'il convient peut-être d'interpréter deux procédures rituelles mises en cuvre par les meurtriers soucieux d'éviter la colère en retour de leur victime. Dans un cas, il s'agit de lécher le sang coulant des blessures et de le recracher dans la bouche du mort; dans l'autre, le meurtrier moissonne sur le cadavre les extrémités : mains, nez, oreilles 
de manière à en faire un collier autour de ses aisselles ou bien afin de les déposer sur le corps de sa victime (Rohde, 1952 : 299-603; Gotsmich, $1955: 349-366$; Vian, $1981: 167$ ). Cette derniere pratique, dite maschalismos, porte le même nom que l'offrande faite aux dieux, dans les sacrifices de victimes animales, de petits morceaux de chair crue prélevés sur toute la surface et placés ensuite sur les cuisseaux mis à brûler sur l'autel. Ces maschalismata, déjà attestés par des lexicographes anciens, et maintenant confirmés par une loi sacrée de l'Attique (Vanderpool, 1970), sont homologues à ces prémices, apargmata, offertes par le meurtrier léchant le sang de sa victime et le crachant dans la bouche du cadavre, ainsi traité, semble-t-il, dans l'un et l'autre cas, comme une victime d'un sacrifice et non d'un meurtre. Usant de ce biais rituel qui transformait en victime sacrificielle le cadavre de sa victime, le meurtrier pouvait ainsi ne pas quitter l'espace sacrificiel d'où le sang versé d'un être humain devait aussitôt l'exclure en le vouant à la fuite hallucinée sinon à l'exil perpétuel.

C'est sur Oreste que se condensent les représentations les plus vives des liens se nouant entre une victime et son meurtrier dans le temps même où semble s'imposer la vision de l'homicide abstrait de ce conglomérat de souillures, de solidarités et de rituels de purification qui semble appartenir à un avant de Dracon et des tribunaux du sang, dans l'espace nouveau de la cité. A cet imaginaire enté sur Oreste, il faut ajouter la dimension du meurtrier valorisé et se traitant lui-même dont certaines figures ne sont pas étrangères aux récits mêmes de matricide affolé. Au lieu de se laisser prendre dans le filet des liens effrayants et convulsifs dont le meurtrier ne pourra s'échapper qu'au terme d'un long et dangereux parcours rituel, il est permis à l'homicide de neutraliser immédiatement les effets du sang versé en coupant brutalement tous ses liens avec son territoire et son groupe social. Le meurtrier peut s'exiler, et, ce faisant, il se transforme sur le champ en mort-vivant : sans droits, privé de sa maison, de ses biens, de ses rapports avec les dieux. Un mort vivant qui laisse derrière lui la violence et le pouvoir d'un "nouveau mort" en même temps que son ancien territoire. Un mort vivant en sursis, le temps de gagner le sanctuaire de Delphes, ce lieu extraterritorial, et d'obtenir du maître de l'oracle l'autorisation de partir en quête d'un territoire nouveau, de 
s'en aller à la recherche d'un espace où s'installer, habiter, fonder une cité nouvelle sans laquelle il ne peut y avoir de maison ni de vie sociale. C'est à Delphes, et seulement à Delphes, que les meurtriers choisissant de s'exiler peuvent rencontrer le dieu, lui-même homicide et naguère exilé, qui leur ouvre la voie d'une territorialisation sans passé, sans mémoire ni du sang versé ni des liens anciens. L'ex-meurtrier se transforme à la manière d'Apollon en Fondateur destiné à devenir après sa mort le glorieux Archégète en qui la cité nouvelle reconnaît son Ancêtre commun et immédiat. Meurtriers plus imaginaires que réels (au moins, dans notre documentation d'après coup), mais qui viennent dire, dans la Grèce de la colonisation entre le VIIIème et le Vème siècle av. notre ère, les vertus inaugurales de l'homicide qui sait se purifier en se séparant radicalement de sa première identité. Individu pratiquant l'auto-fondation sur un mode "politique", à la manière grecque d'une cité autonome et qui peut s'implanter sur n'importe quelle partie de la peau de la terre.

M. Detienne E.P.H.E.

\section{Références bibliographiques}

\section{AUTEURS GRECS :}

Eschyle, Euménides, éd. Paul Mazon, Paris, Les Belles Lettres, 1920

Eschyle, Choéphores, éd. Paul Mazon, Paris, Les Belles Lettres, 1920

Héraclite, Fragments, éd. J. Bollack et H. Wissman, Paris, Editions de Minuit, 1972

Homère, Odyssée, éd. V. Bérard, Paris, Les Belles Lettres, 1933

Iliade, éd. Paul Mazon, Paris, Les Belles Lettres, 1937-38 
Hippocrate, Sur la maladie sacrée, éd. Littré.

Pausanias, Description de la Grèce, I-IV, éd. W.H.S. Jones, Collection Loeb, Harward University Press, 1918-1935

Pindare, Pythiques, éd. A. Puech, Les Belles Lettres, 1922-23

Platon, Lois, éd. des Places, Paris, Les Belles Lettres

Stésichore, Orestie, PMG.

\section{AUTRES RÉFÉRENCES:}

Delcourt, Marie

1959 Oreste et Alcméon. Étude sur la projection légendaire du matricide en Grèce, Paris, Les Belles Lettres.

Detienne, Marcel

1990 "Qu'est-ce qu'un site ?", et "Apollon Archégète. Un modèle politique de la territorialisation", dans Tracés de fondation, M. Detienne éd., Paris-Louvain, Peeters, 1-16 et 301-311.

1994 "Retour sur la bouche de la Vérité" dans M. Detienne, Les maîtres de Vérité dans la Grèce archaïque, Paris, Pocket-Agora, pp. 5-31 (1ère éd. 1967).

Durand, Jean-Louis

1990 "Formules attiques du fonder", dans Tracés de fondation, M. Detienne éd., Paris-Louvain, Peeters, pp. 271-287

Gernet, L.

1955 "Delphes et la pensée religieuse en Grèce", Annales E. S. C., 526-542.

1984 "Le droit pénal de la Grèce ancienne", dans Du châtiment dans la cité. Supplices corporels et peine de mort dans le monde antique, Rome, Palais Farnèse, pp. 9-35.

Gotsmich, A.

1955 "Der Mashalismos und seine wiedergabe in der griechischen Kunst", Feskgabe für B. Kraff, pp. 349-366

Moulinier, L.

1952 Le pur et l'impur dans la pensée des Grecs, Paris, Klincksieck 
Neschke, A.

1986 "L'Orestie de Stésichore et la tradition littéraire du mythe des Atrides avant Eschyle", L'antiquité classique, 55, pp. 283-301.

Parker, Robert

1983 Miasma. Pollution and Purification in early Greek Religion, Oxford, Clarendon Press

Rohbe, R.

1893 (1952) Psychë, tr. fr. A. Reymond, Paris, Payot, pp. 599-603

Van Effenterre, H. et Ruzé, F.

1994 Nomima. Recueil d'inscriptions politiques et juridiques de l'archaisme grec, I, Rome, Palais Farnèse.

Vanderpool, E.

1970 "A Lex sacra of the attic Deme Phrearrhioi", Hesperia, pp. 47-53.

Vian, F.

1981 "Commentaires à Apollonios de Rhodes", Argonautiques, IV, t. III, Paris, Les Belles Lettres 